\title{
Flow cytometry as tool to monitor chain elongation performance
}

Kevin Sabbe*,a,b, Sylvia Gildemyn ${ }^{b}$, Frederiek-Maarten

Kerckhofa, Nico Boon ${ }^{\text {, }}$, Ramon Ganigué ${ }^{a}$

* presenter, Kevin.Sabbe@UGent.be

a Ghent University, Belgium; ${ }^{b}$ OWS nv, Belgium

\section{HIGHLIGHTS:}

- Flow cytometry is a rapid and reliable method to monitor the microbial community in a chain elongating reactor

- Phenotypic fingerprints of different reactor states can be differentiated via flow cytometry.

- A reactor microbiome for lactic acid chain elongation, disturbed due to caproic acid toxicity, has the potential to return to its stable state during recovery.

BACKGROUND: During the past years there has been a growing awareness that we need to strive to a circular bio economy, which has stimulated the development of novel biological processes for the production of added-value platform chemicals from organic waste streams. These new developments go hand in hand with an increasing need for process stability and performance insurance. Therefore, a good monitoring strategy is essential. The state-of-the-art mainly relies on monitoring physicochemical input and output parameters. Here we report the development of a novel monitoring strategy in which microbial community (MC)-parameters play a key role. The MC-parameters are obtained via flow cytometry (FCM), which is a MC analysis technique that allows a fast assessment of its phenotypical diversity $[1,2]$. FCM as a process monitoring and control tool has mostly been applied to the $\mathrm{MC}$ in environments containing little nutrients and lower microbial abundance, such as drinking water [3]. More recently, the use of FCM is emerging for the analysis of the MC and the detection of disturbances through flow cytometric fingerprinting $[3,4]$.

In this study, FCM is studied as a key tool for the fast detection of community changes and applied to caproic acid (CA) production via lactic acid (LA) chain elongation, with the potential of applying this on other fermentation processes in the future. We operated mixed-culture CSTRbioreactors that were fed with a synthetic medium that contains LA (20.8 $\mathrm{g} / \mathrm{L}$ ) as main carbon source. During reactor operation, several physicochemical parameters $(\mathrm{pH}$, electroconductivity, biogas yield and composition, carboxylate yields and spectrum, etc.) were monitored along with the microbiology. From the FCM data, the cell counts and diversity parameters were derived. The community structure was determined as a phenotypic fingerprint (PFP) based on the identification of phenotypes with a model-based approach based on Gaussian Mixture Models (GMM) [5]. 
RESULTS \& DISCUSSION : During reactor operation, periods of decreased performance or process failure were observed. The results of one period of process failure are presented. Physiochemically, process failure was initially observed as a sudden, complete stop of the gas production, while prior to this event, around 1.2 Lbiogas. Lreactor $^{-1}$. $\mathrm{d}^{-1}$ containing $23.25 \% \mathrm{H}_{2}$ and $73.75 \%$ $\mathrm{CO}_{2}$ was produced. $\mathrm{H}_{2}$ and $\mathrm{CO}_{2}$ are by-products of LA chain elongation [69]. Analysis of the carboxylate spectrum showed the highest $C A$ concentration $(8.0 \mathrm{~g} / \mathrm{L})$ observed in the reactors at the moment of process failure. LA accumulated up to $8.4 \mathrm{~g} / \mathrm{L}$ and the CA concentration decreased. Activity resumed once the CA-concentration had decreased to $3.2 \mathrm{~g} / \mathrm{L}$. It is therefore hypothesised that CA-toxicity was a possible cause of process failure in the process under study.

A GMM-model was generated to determine the PFP of the reactor samples. Clear shifts in the community structure based on the PFP could be observed during this period (figure $1, a$ ). Ordination of the samples based on the Bray-Curtis dissimilarities via Canonical Correspondence Analysis (CCA) shows the evolution of the reactor samples during normal operation, process failure and recovery (figure $1, \mathrm{~b}$ ). Samples taken prior to process failure cluster together in the CCA plot, whereas the samples taken during process failure and recovery are ordinated in a counterclockwise pattern. The further in the recovery phase, the closer the samples are ordinated to the plotted to the cluster of samples from before process failure. This indicates that the microbiome performing LA chain elongation can return to its stable state prior to the crash.
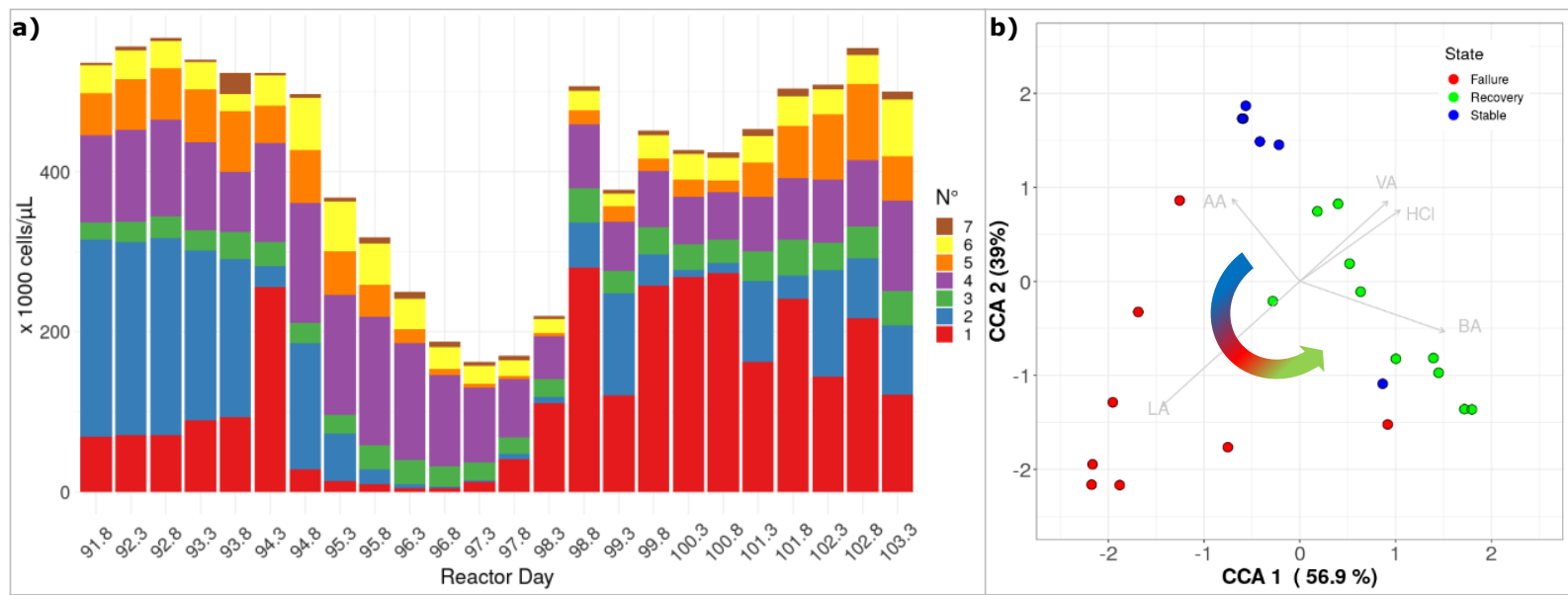

Figure 1. a) absolute phenotypic distribution over time, based on a GMMmodel with seven identified phenotypes. b) CCA analysis of the samples based on the Bray-Curtis dissimilarities, constrained by the LA, acetic acid $(\mathrm{AA})$, butyric acid (BA) and valeric acid (VA) concentrations, and the $\mathrm{HCl}$ requirements for $\mathrm{pH}$ control.

CONCLUSION: FCM is a suitable technique for fast determination of the PFP of a mixed-culture microbiome. Changes in reactor performance can be monitored as well on microbial level. FCM is therefore a promising tool to be included into the monitoring strategy of LA chain elongation, with the potential of extending its application on other bioproduction processes. 


\section{REFERENCES:}

1. Park, H.-S, Schumacher, R., Kilbane II, J.J., New method to characterize microbial diversity using flow cytometry, Journal of Industrial Microbiology \& Biotechnology, 32, 94-102

2. Props, R., Monsieurs, P., Mysara, M., Clement, L., Boon, N., Measuring the biodiversity of microbial communities by flow cytometry., Methods in Ecology and Evolution, 7, 1376-1358

3. Props, R., Rubbens, P., Besmer, M., Buysschaert, B., Sigrist, J., Weilenmann, H., Waegemann, W., Boon, N., Hammes, F., Detection of microbial disturbances in a drinking water microbial community through continuous acquisition and advanced analysis of flow cytometry data., Water Research, 145, 73-82

4. Buysschaert, B., Kerckhof, F.-M., Vandamme, P., De Baets, B., Boon, N., Flow cytometric fingerprinting for microbial strain discrimination and physiological characterization., Cytometry Part A, 93, 201-212

5. Rubbens, P., Props, R., Kerckhof, F.-M., Boon, N., \& Waegeman, W., PhenoGMM: Gaussian mixture modelling of microbial cytometry data enables efficient predictions of biodiversity., BioRxiv, 641464

6. de Araújo Cavalvante, W., Carrhá Leitão, R, Gehring, T.A., Angenent, L.T., Tédde Santealla, S., Anaerobic Fermentation for N-Caproic Acid Production: A Review., Process Biochemistry, 54, 106-119

7. De Groot, V., Coma, M., Arnot, T., Leak, D.J., Lanham, A.B., Medium Chain Carboxylic Acids from Complex Organic Feedstocks by Mixed Culture Fermentation., Molecules, 24, 398

8. Kucek, L.A., Xu, J., Nguyen, M., Angenent, L.T., Conversion of Ilactate into $\mathrm{n}$-caproate by a continuously fed reactor microbiome., Water Research, 93, 163-171

9. Zhu, X., Zhou, Y., Wang, Y., Wu, T., Li, X., Li, D., Tao, Y., Production of High-Concentration n-Caproic Acid from Lactate through 